\title{
Accuracy and Anatomical Coverage of Perfusion CT Assessment of the Blood-Brain Barrier Permeability: One Bolus versus Two Boluses
}

\author{
Jan Willem Dankbaar ${ }^{a, c}$ Jason Hom ${ }^{a}$ Thomas Schneider ${ }^{d}$ Su-Chun Cheng ${ }^{b}$ \\ Benison C. Lau ${ }^{a}$ Irene van der Schaafc Sunny Virmani ${ }^{d}$ Scott Pohlman ${ }^{d}$ \\ William P. Dillon ${ }^{a}$ Max Wintermark ${ }^{a}$ \\ ${ }^{a}$ Neuroradiology Section, Department of Radiology, and ${ }^{b}$ Department of Epidemiology and Biostatistics, \\ University of California, San Francisco, Calif., USA; ' Department of Radiology, University Medical Center Utrecht, \\ Utrecht, and ${ }^{\mathrm{d}} \mathrm{CT}$ Clinical Science Group, Philips Medical Systems, Best, The Netherlands
}

\section{Key Words}

Perfusion computed tomography $\cdot$ Blood-brain barrier

permeability $\cdot$ Patlak model

\begin{abstract}
Purpose: To assess whether blood-brain barrier permeability (BBBP) values, extracted with the Patlak model from the second perfusion CT (PCT) contrast bolus, are significantly lower than the values extracted from the first bolus in the same patient. Materials and Methods: 125 consecutive patients (29 with acute hemispheric stroke and 96 without stroke) who underwent a PCT study using a prolonged acquisition time up to 3 min were retrospectively identified. The Patlak model was applied to calculate the rate of contrast leakage out of the vascular compartment. Patlak plots were created from the arterial and parenchymal time enhancement curves obtained in multiple regions of interest drawn in ischemic brain tissue and in nonischemic brain tissue. The slope of a regression line fit to the Patlak plot was used as an indicator of BBBP. Square roots of the mean squared errors and correlation coefficients were used to describe the quality of the linear regression model. This was performed separately for the first and the second PCT
\end{abstract}

bolus. Results from the first and the second bolus were compared in terms of BBBP values and the quality of the linear model fitted to the Patlak plot, using generalized estimating equations with robust variance estimation. Results: BBBP values from the second bolus were not lower than BBBP values from the first bolus in either nonischemic brain tissue [estimated mean with 95\% confidence interval: 1.42 (1.101.82) $\mathrm{ml} \cdot 100 \mathrm{~g}^{-1} \cdot \mathrm{min}^{-1}$ for the first bolus versus $1.64(1.31-$ 2.05) $\mathrm{ml} \cdot 100 \mathrm{~g}^{-1} \cdot \mathrm{min}^{-1}$ for the second bolus, $\mathrm{p}=1.00$ ] or in ischemic tissue [1.04 (0.97-1.12) $\mathrm{ml} \cdot 100 \mathrm{~g}^{-1} \cdot \mathrm{min}^{-1}$ for the first bolus versus $1.19(1.11-1.28) \mathrm{ml} \cdot 100 \mathrm{~g}^{-1} \cdot \mathrm{min}^{-1}$ for the second bolus, $p=0.79$ ]. Compared to regression models from the first bolus, the Patlak regression models obtained from the second bolus were of similar or slightly better quality. This was true both in nonischemic and ischemic brain tissue. Conclusion: The contrast material from the first bolus of contrast for PCT does not negatively influence measurements of BBBP values from the second bolus. The second bolus can thus be used to increase anatomical coverage of BBBP assessment using PCT.

Copyright $\odot 2008$ S. Karger AG, Basel

S.P. and S.V. are employees of Philips Medical Systems. T.S. was an employee of Philips Medical Systems at the time of this study.

\section{KARGER}

(C) 2008 S. Karger AG, Basel

Fax +41613061234 E-Mail karger@karger.ch www.karger.com
Accessible online at: www.karger.com/ced
Max Wintermark, MD, Department of Radiology, Neuroradiology Section

University of California, San Francisco, 505 Parnassus Avenue, Box 0628

San Francisco, CA 94143-0628 (USA)

Tel. +1 415353 1668, Fax +1 4153538593

E-Mail Max.Wintermark@radiology.ucsf.edu 


\section{Introduction}

Brain perfusion CT (PCT) is currently used for the evaluation of patients with symptoms of acute stroke [1, 2]. PCT allows to distinguish between infarct core and brain tissue at risk [3-7]. More recently, PCT data were also used to characterize the blood-brain barrier permeability (BBBP) [8-10], in an effort to predict which stroke patients are going to develop hemorrhagic transformation.

A well-known limitation of PCT is its limited anatomical coverage, typically $2-4 \mathrm{~cm}$. One of the approaches to overcome this limitation is by scanning at two consecutive levels with two separate contrast bolus injections [11]. In terms of BBBP measurements, it was suggested in a recent study [9] that the contrast material from the first bolus could saturate the interstitial compartment, by extravasating from the vascular to the interstitial compartment. Theoretically this would impede further extravasation of contrast during the second bolus. BBBP values calculated from the second bolus would then turn out to be lower than they are in reality.

The goal of this study was to assess, in stroke and nonstroke patients, whether BBBP values extracted with the Patlak model from the second PCT contrast bolus are lower than the values extracted from the first bolus.

\section{Methods}

\section{Design}

Imaging data obtained as part of standard clinical stroke care at our institution were retrospectively reviewed with the approval of the institutional review board. At our institution, patients with suspicion of acute stroke and no history of significant renal insufficiency or contrast allergy routinely undergo a stroke CT survey including: noncontrast CT of the brain, PCT at two crosssectional positions, CT angiogram of the cervical and intracranial vessels, and postcontrast cerebral CT, obtained in this chronological sequence.

We retrospectively identified a consecutive series of $130 \mathrm{pa}-$ tients admitted to the UCSF Medical Center from January 2007 to June 2007 who met the following inclusion criteria: (a) admission to the emergency room with signs and symptoms suggesting hemispheric stroke within $12 \mathrm{~h}$ after symptom onset and (b) no evidence of intracerebral hemorrhage on the admission noncontrast CT. Patients' charts were reviewed for demographic and clinical data, as well as for the interpretation of imaging studies obtained during the hospital stay and discharge diagnosis.

\section{Imaging Protocol}

Each PCT study involved successive gantry rotations performed in cine mode during intravenous administration of iodinated contrast material. Images were acquired and recon- structed at a temporal sampling rate of 1 image/s for the first $45 \mathrm{~s}$. Additional gantry rotations were obtained at $60,90,120$, 150 and $180 \mathrm{~s}$. Acquisition parameters were $80 \mathrm{kVp}$ and 100 $\mathrm{mAs}$. Two successive PCT series at two different levels were performed following the noncontrast $\mathrm{CT}$ and prior to the $\mathrm{CT}$ angiogram. The first PCT series was obtained at the level of the third ventricle and the basal ganglia, and the second PCT series above the lateral ventricles. For each PCT series, a $40-\mathrm{ml}$ bolus of iohexol (Omnipaque, Amersham Health, Princeton, N.J., USA; $300 \mathrm{mg} / \mathrm{ml}$ of iodine) was administered into an antecubital vein using a power injector at an injection rate of $5 \mathrm{ml} / \mathrm{s}$ for all patients. The interval between both bolus injections was approximately 4 min. CT scanning was initiated $7 \mathrm{~s}$ after the start of the injection of the contrast bolus.

\section{Image Postprocessing}

PCT data were analyzed utilizing PCT software developed by Philips Medical Systems (Cleveland, Ohio, USA). This software relies on the central volume principle, which is the most accurate for low injection rates of iodinated contrast material [12]. After motion correction and noise reduction by an anisotropic, edgepreserving spatial filter, the software applies curve fitting by least mean squares to obtain mathematical descriptions of the timedensity curves for each pixel. A closed-form (noniterative) deconvolution is then applied to calculate the mean transit time (MTT) map [13]. The deconvolution operation requires a reference arterial input function (most often within the anterior cerebral artery), automatically selected by the PCT software within a region of interest (ROI) drawn by the user. The cerebral blood volume $(\mathrm{CBV})$ map is calculated from the area under the time-density curves [14]. The PCT infarct core and brain tissue at risk are automatically calculated by the software using CBV and MTT thresholds reported in the literature as the most accurate (PCT tissue at risk: MTT $>145 \%$ of the contralateral side values, CBV $\geq 2.0 \mathrm{ml} \cdot 100 \mathrm{~g}^{-1}$; PCT infarct core: MTT $>145 \%$ of the contralateral side values, $\mathrm{CBV}<2.0 \mathrm{ml} \cdot 100 \mathrm{~g}^{-1}$ ) [5].

BBBP measurements were extracted from PCT data using a second, prototype software developed by Philips Medical Systems. This software is based on the Patlak model [15], which is described in detail in the appendix. Applying the Patlak model to PCT involves performing a linear regression using data calculated from the delayed phase (not first-pass) of the PCT data sets [9]. The slopes of the acquired regression lines are used as an indicator of BBBP. Two variables measuring the quality of the linear fit were used to assess whether the PCT data from the second bolus met the assumptions of the Patlak model just as well as PCT data from the first bolus: (1) the square roots of the mean squared errors are a measure of variability around a straight line, i.e. a value close to 0 indicates a smaller spread of data points around the line, corresponding to a better fit; (2) the correlation coefficients (R) measure the strength of a linear relationship, i.e. an $\mathrm{R}$ value closer to 1 indicates a stronger linearity.

Slopes, square roots of the mean squared errors $(\sqrt{ } \mathrm{MSE})$ and correlation coefficients (R) were measured in ROIs corresponding to vascular territories, drawn on each PCT slice. For the purpose of the statistical analysis, ROIs located within nonischemic brain parenchyma (both in stroke and nonstroke patients) were distinguished from ROIs in ischemic brain parenchyma (i.e. infarct core and tissue at risk, only present in stroke patients). 
Table 1. Patient demographics

\begin{tabular}{lll}
\hline & $\begin{array}{l}\text { Stroke } \\
\text { patients }\end{array}$ & $\begin{array}{l}\text { Nonstroke } \\
\text { patients }\end{array}$ \\
\hline Patients & 29 & 96 \\
Men & $12(43 \%)$ & $42(44 \%)$ \\
Age, years & 72 & 55 \\
$\quad$ Median & $66-82$ & $45-70$ \\
$\quad$ Interquartile range & $52-93$ & $17-91$ \\
$\quad$ Range & 3 & 3.5 \\
Time from stroke to PCT, h & $2-6$ & $2-7$ \\
$\quad$ Median & $1.5-12$ & $1-12$ \\
$\quad$ Interquartile range & & \\
$\quad$ Range & 3 & \\
Stroke location & 24 & \\
$\quad$ ACA and MCA territories & 2 & \\
$\quad$ MCA territory & 0 & \\
ACA territory & & \\
$\quad$ PCA territory & $32.4 \pm 38.5$ & \\
Admission infarct core volume, $\mathrm{cm}^{3}$ & & \\
$\quad$ Mean \pm SD & $0.2-146.6$ & \\
Range & & \\
Final infarct volume, cm & & \\
Mean \pm SD & $97.5 \pm 94.3$ & \\
Range & $3.8-311.4$ & \\
Admission tissue at risk volume, $\mathrm{cm}^{3}$ & & \\
$\quad$ Mean \pm SD & $56.0 \pm 43.4$ & \\
Range & $0.8-15.3$ & \\
\hline
\end{tabular}

$\mathrm{ACA}=$ Anterior cerebral artery; $\mathrm{MCA}=$ medial cerebral $\mathrm{ar}-$ tery; $\mathrm{PCA}=$ posterior cerebral artery.

\section{Statistical Analysis}

The 3 parameters - slopes, $\sqrt{ }$ MSE and R - extracted from PCT data from the first and the second bolus in ischemic and nonischemic ROIs, were compared using generalized estimating equation models with robust variance estimation. The tested null hypotheses were that BBBP measurements (slopes) were not lower for the second bolus compared to the first bolus, and that linear regression fitting was not worse (higher $\sqrt{ }$ MSE and lower $\mathrm{R}$ ) for the second bolus compared to the first. Because the distributions of the 3 mentioned parameters were not normal but skewed, estimated mean values were reported rather than simple means. For all values, 95\% confidence intervals were also calculated.

\section{Results}

\section{Patients and Imaging Studies}

One hundred and thirty patients matched our inclusion criteria; 29 of these had an acute ischemic hemispheric stroke, and 101 did not have any abnormalities on their PCT studies (nonstroke patients). Final diagnoses in nonstroke patients were: transient ischemic attacks in 12 ,
Table 2. Estimated means and $95 \%$ confidence intervals (in parentheses) for the BBBP measurements according to the Patlak model, and the parameters describing the quality of the linear regression $(\sqrt{ }$ MSE, R), both for the first bolus (bolus 1$)$ and the second bolus (bolus 2), in ischemic and nonischemic ROIs

\begin{tabular}{|c|c|c|c|}
\hline & Bolus 1 & Bolus 2 & $\begin{array}{l}\mathrm{p} \\
\text { value }\end{array}$ \\
\hline \multicolumn{4}{|l|}{ Ischemic ROIs } \\
\hline BBBP & $1.42(1.10-1.82)$ & $1.64(1.31-2.05)$ & 0.79 \\
\hline \multicolumn{4}{|c|}{ Quality of linear regression } \\
\hline$\sqrt{\mathrm{MSE}} \cdot 10^{4}$ & $0.96(0.74-1.24)$ & $0.73(0.48-1.13)$ & 0.87 \\
\hline $\mathrm{R}$ & $0.69(0.58-0.80)$ & $0.79(0.68-0.90)$ & 0.90 \\
\hline \multicolumn{4}{|c|}{ Nonischemic ROIs } \\
\hline BBBP & $1.04(0.97-1.12)$ & $1.19(1.11-1.28)$ & 1.0 \\
\hline \multicolumn{4}{|c|}{ Quality of linear regression } \\
\hline$\sqrt{\mathrm{MSE}} \cdot 10^{4}$ & $0.42(0.35-0.49)$ & $0.41(0.33-0.49)$ & 0.63 \\
\hline $\mathrm{R}$ & $0.84(0.81-0.87)$ & $0.87(0.84-0.90)$ & 0.99 \\
\hline
\end{tabular}

The $\sqrt{\text { MSE }}$ is a measure of variability around a straight line: a value close to 0 indicates a smaller spread of data points around the line, corresponding to a better fit. The correlation coefficient $\mathrm{R}$ measures the strength of a linear relationship: an $\mathrm{R}$ value closer to 1 indicates stronger linearity.

vertigo in 9 , migraine in 7 , other neurological disorder in 9 , adverse drug reaction in 3 , other nonneurological disorders in 12, undetermined in 49. In these 101 patients, the absence of hemispheric, ischemic stroke or major hemispheric abnormality was confirmed on follow-up CT or MRI, and clinical workup. Five patients were excluded from the nonstroke group, because of PCT data that could not be analyzed due to motion artifacts or improper timing of contrast bolus injection. The patient characteristics of the 29 stroke and the 96 nonstroke patients are summarized in table 1.

The radiation dose for the prolonged PCT acquisition (cine mode over $45 \mathrm{~s}$, then additional gantry rotations at $60,90,120,150$ and $180 \mathrm{~s})$ was only $10 \%(0.25 \mathrm{mSv})$ higher than the dose for the conventional PCT protocol (cine mode over $45 \mathrm{~s}$ ).

The Patlak analyses for the 29 stroke and 96 nonstroke patients were performed in a total number of 504 ischemic ROIs and 2,194 nonischemic ROIs.

BBBP Measurements and Parameters Describing the Quality of the Linear Regression according to the

Patlak Model

BBBP values were calculated from the slope of the regression line fit to the Patlak plot (table 2, fig. 1). BBBP 
Fig. 1. Example of graphical illustration of $\mathrm{BBBP}$, calculated from the slope of a regression line fit to the Patlak plot. a PCT infarct core and tissue at risk threshold maps. b BBBP color maps; BBBP values from the second bolus (bolus 2) were not lower than the values from the first bolus (bolus 1). c Patlak plots that were constructed from arterial and parenchymal time enhancement curves, as explained in the appendix, in an infarcted tissue ROI and a nonischemic tissue ROI. These Patlak plots illustrate how small the differences in slope (BBBP) and linearity between bolus 1 and 2 are. Of note, the slope of the plot from the infarcted tissue ROI is steeper than that of the nonischemic tissue ROI.
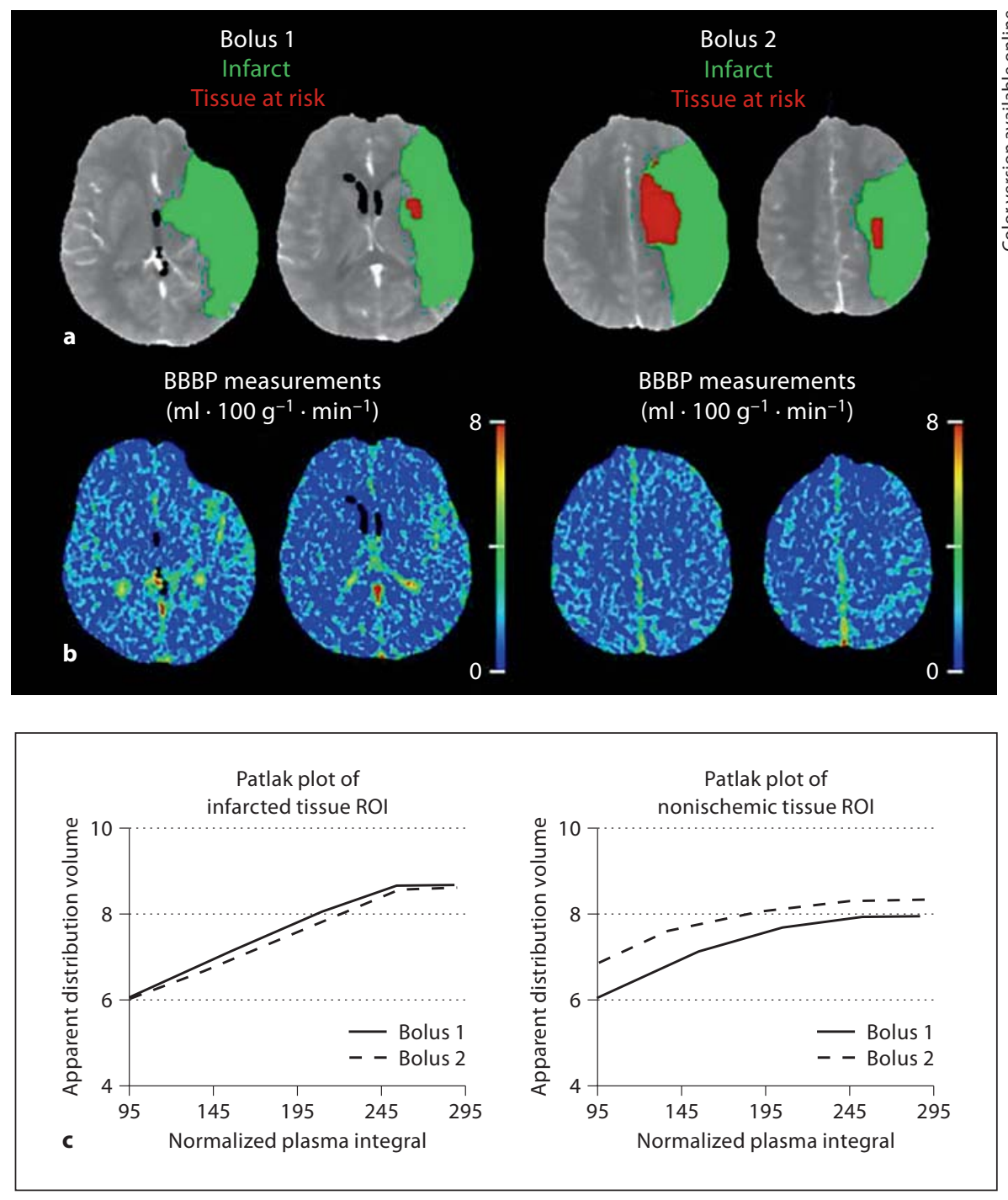

values from the second bolus were not significantly lower than values calculated from the first. This was true both in ischemic ( $p=0.79)$ and nonischemic ROIs $(p=1.00)$.

The quality of the linear regression, as part of the application of the Patlak model to the PCT data for BBBP measurement, was expressed in $\sqrt{\text { MSE }}$ and R (table 2). $\sqrt{M S E}$ values from the second bolus were not higher (variability around the regression line was not worse) than values from the first. This was true both in ischemic ROIs $(\mathrm{p}=0.87)$ and in nonischemic ROIs $(\mathrm{p}=0.63)$. Correlation coefficients $\mathrm{R}$ from the second bolus were not lower (linearity was not worse) than coefficients from the first bolus. This was true both in ischemic ROIs $(p=0.90)$ and in nonischemic ROIs $(\mathrm{p}=0.99)$.

\section{Discussion}

Recently, there has been an interest in using PCT imaging to assess $\mathrm{BBBP}$ alterations that may be a predisposing condition for hemorrhagic transformation in acute stroke patients $[8,10]$. In the pursuit of this objective, whole-brain coverage would be desirable, which in the case of PCT is achieved by obtaining 2 successive PCT series with 2 successive injections of a contrast bolus. However, a recent study [9] suggested that the contrast material from the first bolus could saturate the interstitial compartment, by extravasating from the vascular compartment to the interstitial compartment. Theoretically this would impede further extravasation of contrast during the second bolus. 
In this study, we demonstrated that the contrast material injected for the first bolus does not interfere with the BBBP measurements from the second bolus. BBBP measurements from the second bolus were not lower than BBBP values measured from the first. The assumptions of the Patlak model are met equally well by the two boluses. This was true both for the ischemic and for the nonischemic brain tissue. The calculation of Patlak plots and BBBP values was derived exclusively from the delayed phase of the PCT acquisition, as this was demonstrated to be the most robust approach [9].

We acknowledge several limitations to our study. For evident ethical reasons related to radiation dose, it was not possible to image the same levels twice with the two consecutive PCT contrast boluses. Instead, we decided to compare two boluses obtained at two different levels of the brain using generalized estimating equation models with robust variance estimation, to account for withinpatient correlations in addition to fixed effects for the two boluses, ischemic/nonischemic ROIs and their interaction. The different locations of the two PCT series could have influenced the amount of ischemic brain tissue in each of them (because of differences e.g. in gray-white matter ratio, hemodynamics or collaterals), which in turn could have impacted the comparison between the two boluses in terms of BBBP values. In any case, the quality of the linear regression required by the Patlak model was not different for the two boluses, and the values from the second bolus were not lower than those from the first, both in the nonischemic and in the ischemic brain parenchyma.

Finally, we limited our analysis to the Patlak model. The goal of this study was to evaluate how the Patlak model could be applied to PCT data. We did not assess other models that have been applied to PCT data to calculate BBBP, such as the distributed parameter model $[8$, 16]. Future studies are needed to determine whether our conclusions regarding the acceptable use of a second PCT bolus hold for these alternative models.

In conclusion, the contrast material from the first bolus of contrast for PCT does not negatively influence measurements of BBBP values from the second bolus. The second bolus can thus be used to increase anatomical coverage of BBBP assessment using PCT. Further research is required to investigate the relevance of these BBBP measurements in terms of their predictive value for hemorrhagic transformation in acute stroke patients.

\section{Appendix}

The model described by Patlak et al. [15] and Patlak and Blasberg [17] is a theoretical model of blood-brain exchange. It is a multicompartmental model that assumes the unidirectional transfer of a tracer from a reversible (arterial) compartment to an irreversible tissue compartment (in this case the brain parenchyma) for a certain period of time. Transfer of tracer is assumed to be unidirectional when a steady-state phase is reached between reversible compartments (intravascular space and the bloodbrain barrier complex). Such a steady-state phase can only occur after the initial, rapid changes in tracer concentration have subsided so that the arterial concentration decreases slowly enough for the tissue compartment to follow.

The graphical representation of the Patlak model is called Patlak plot (fig. 1). When the Patlak plot is linear, unidirectional transfer is said to be present. The slope of the plot indicates the rate of transfer between the reversible and the irreversible compartments. When applied to PCT, the Patlak model uses iodinated contrast medium as the tracer and takes advantage of the Hounsfield density to be directly proportional to the iodinated contrast concentration. Enhancement within the arterial input function (reversible compartment) and within the parenchyma (irreversible compartment) over time is used to construct the Patlak plot. The plot is described by the following equation:

$$
T t / A t=K i \cdot\left(\int_{0}^{t} A d t\right) / A t+V
$$

In this equation, $T t$ is the tissue enhancement at time $t ; A t$ is the arterial enhancement at time $t ; K i$ is the rate constant of net contrast transfer; $\int A d t$ is the integral along time of arterial concentration, and $V$ is the distribution volume, which is typically considered to be equal to the $\mathrm{CBV}$ in the considered ROI. The ratio of $T t$ to $A t$, which is plotted on the y-axis, is called 'apparent distribution volume'. The ratio of the integral of $A$ to $A t$, which is plotted on the x-axis, is called 'normalized plasma integral'. The slope of a regression line fit to the linear part of the Patlak plot is an approximation of $K i$ (the rate of transfer) at that time. This value represents the amount of accumulated tracer in relation to the amount of tracer that has been available in plasma, and is a measurement of BBBP expressed in milliliters per $100 \mathrm{ml}$ per minute. The y-axis intercept is equal to the CBV.

\section{Acknowledgements}

This project was supported by a grant from Philips Medical Systems.

M.W. receives funding from the National Center for Research Resources, grant KL2 RR024130, GE Healthcare, Philips Medical Systems and Boston Scientific; he is a consultant for Concentric and Mitsubishi Pharma. The content of the article is solely the responsibility of the authors and does not necessarily represent the official views of the National Institute of Neurological Disorders and Stroke, the National Center for Research Resources, the National Institutes of Health or the other sponsors. 


\section{References}

1 Fisher M: The ischemic penumbra: identification, evolution and treatment concepts. Cerebrovasc Dis 2004;17(suppl 1):1-6.

$\checkmark 2$ Meuli RA: Imaging viable brain tissue with CT scan during acute stroke. Cerebrovasc Dis 2004; 17(suppl 3):28-34.

>3 Nabavi DG, Dittrich R, Kloska SP, Nam EM, Klotz E, Heindel W, Ringelstein EB: Window narrowing: a new method for standardized assessment of the tissue at risk maximum of infarction in CT based brain perfusion maps. Neurol Res 2007;29:296-303.

4 Kloska SP, Nabavi DG, Gaus C, Nam EM, Klotz E, Ringelstein EB, Heindel W: Acute stroke assessment with CT: do we need multimodal evaluation? Radiology 2004;233: 79-86.

5 Wintermark M, Flanders AE, Velthuis B, Meuli R, van Leeuwen M, Goldsher D, Pineda C, Serena J, van der Schaaf I, Waaijer A, Anderson J, Nesbit G, Gabriely I, Medina V, Quiles A, Pohlman S, Quist M, Schnyder P, Bogousslavsky J, Dillon WP, Pedraza S: Perfusion-CT assessment of infarct core and penumbra: receiver operating characteristic curve analysis in 130 patients suspected of acute hemispheric stroke. Stroke 2006;37: 979-985.
-6 Parsons MW, Pepper EM, Bateman GA, Wang Y, Levi CR: Identification of the penumbra and infarct core on hyperacute noncontrast and perfusion CT. Neurology 2007; 68:730-736.

7 Schaefer PW, Roccatagliata L, Ledezma C, Hoh B, Schwamm LH, Koroshetz W, Gonzalez RG, Lev MH: First-pass quantitative CT perfusion identifies thresholds for salvageable penumbra in acute stroke patients treated with intra-arterial therapy. AJNR Am J Neuroradiol 2006;27:20-25.

-8 Bisdas S, Hartel M, Cheong LH, Koh TS, Vogl TJ: Prediction of subsequent hemorrhage in acute ischemic stroke using permeability CT imaging and a distributed parameter tracer kinetic model. J Neuroradiol 2007;34:101-108.

9 Dankbaar JW, Hom J, Schneider T, Cheng S, Lau B, van der Schaaf I, Virmani S, Pohlman S, Dillon WP, Wintermark M: Dynamic perfusion-CT assessment of the blood-brain barrier permeability: first-pass versus delayed acquisition. AJNR Am J Neuroradiol, in press.

10 Lin K, Kazmi KS, Law M, Babb J, Peccerelli N, Pramanik BK: Measuring elevated microvascular permeability and predicting hemorrhagic transformation in acute ischemic stroke using first-pass dynamic perfusion CT imaging. AJNR Am J Neuroradiol 2007; 28:1292-1298.
-11 Eastwood JD, Lev MH, Provenzale JM: Perfusion CT with iodinated contrast material. AJR Am J Roentgenol 2003;180:3-12.

$>12$ Wintermark M, Maeder P, Thiran JP, Schnyder P, Meuli R: Quantitative assessment of regional cerebral blood flows by perfusion CT studies at low injection rates: a critical review of the underlying theoretical models. Eur Radiol 2001;11:1220-1230.

13 Axel L: Tissue mean transit time from dynamic computed tomography by a simple deconvolution technique. Invest Radiol 1983; 18:94-99.

14 Ladurner G, Zilkha E, Iliff D, du Boulay GH, Marshall J: Measurement of regional cerebral blood volume by computerized axial tomography. J Neurol Neurosurg Psychiatry 1976;39:152-158.

15 Patlak CS, Blasberg RG, Fenstermacher JD: Graphical evaluation of blood-to-brain transfer constants from multiple-time uptake data. J Cereb Blood Flow Metab 1983;3: $1-7$.

16 Johnson JA, Wilson TA: A model for capillary exchange. Am J Physiol 1966;210:12991303.

$\checkmark 17$ Patlak CS, Blasberg RG: Graphical evaluation of blood-to-brain transfer constants from multiple-time uptake data: generalizations. J Cereb Blood Flow Metab 1985;5:584590. 